The Shape of Knowledge

\title{
Effects of Declarative Information in Instructions for Use
}

\author{
Joyce Karreman \\ Michaël Steehouder \\ University of Twente, Enschede, The Netherlands \\ j.karreman@utwente.nl;m.f.steehouder@utwente.nl
}

\begin{abstract}
Adding declarative information to the essential procedural information in instructions for use might have different effects. Previous research examined the effect of declarative information on the ability to work with the system that is described in the instructions. However, the results of the various experiments were not conclusive. Moreover, other possible effects of declarative information were never investigated before. In the present experiment, the effects of two different types of declarative information on task performance, on cognitive load and on self-efficacy were examined. The results demonstrated that the two types of declarative information negatively affected cognitive load and selfefficacy ratings. The effects on task performance were positive, but moderate.
\end{abstract}

\section{Introduction}

Instructions for use contain different types of information. Procedural information is the most important type of information. It describes the actions that have to be carried out together with the conditions for these actions and the results from these actions. An example of procedural information is: 'Press the left button; the gauge will increase'. All other information is called declarative information. Two different types of declarative information that are found in instructions for use are system information and utilization information. The first type of declarative information explains the internal working of the product; for example 'All functions of the telephone system are stored in the base station which is connected to the different handsets'. Utilization information explains why and under what circumstances the particular functions of the device can be used; for example 'If you assign different numbers to your telephone system, the costs for both numbers can be split up on your bill'.

It is assumed that the different types of declarative information result in a better mental representation of the device and the tasks that have to be carried out (for example [1], [2], [3]). A better, more elaborated mental representation would result in better task performance.
The results of some experiments confirmed these assumptions. Kieras and Bovair [1] demonstrated that system information affected task performance positively. Participants in their experiments who read system information could better operate a fictitious device than participants who only received procedural information. The results of Ummelen's experiment [4] showed that users of instructions that included different types of declarative information could better work with a spreadsheet program than users of instructions that provided only procedural information. However, other experiments did not show effects of declarative information on task performance [5], [6], [7]. Definitely more research is necessary to answer the question whether different types of declarative information positively affect the ability to work with the device or system.

Apart from the possible effect of system information and utilization information on the ability to carry out tasks, these types of declarative information might have other effects. If system and utilization information provoke the formation of a more elaborated mental representation, it might be expected that reading and using instructions with declarative information will require more mental effort than instructions with only procedural information, or in other words: a higher cognitive load will occur [8]. These efforts might be 'paid back' when the user needs the elaborated mental representation to infer lacking elements of the tasks that have to be carried out, because this representation helps to fill the gaps in the procedural knowledge. As far as we know, there is no empirical research that investigated the effect of system information on cognitive load.

Another effect of system and utilization information might lie in the fact that it enhances the confidence of the users that they will be able to solve new and unexpected problems. In Bandura's theory of social learning, this confidence is coined self-efficacy, 'the belief in one's capabilities to organize and execute the sources of action required to manage prospective situations' [9]. Although the concept of selfefficacy is mainly applied to general fields of life such as learning and health, it is possible to apply it on 
more specific situations too, such as mastering a technical device.

\section{Method}

\subsection{Participants}

Sixty-five students of the University of Twente participated in the experiment. They followed one of the social sciences programs (Educational Technology, Business Administration, or Communication studies). They were paid 10 euro for their participation.

\subsection{Materials}

A fictitious device was designed for the experiment; a machine for the production of licorice called GDPC 324. This machine had to be operated by a control panel that was simulated on a computer screen. The panel consisted of a number of buttons and slide controls that could be operated with the mouse, and a display on which feedback was given on the actions.

Three versions of user instructions were written. The first version (P) included only procedural information. It consisted of 11 procedures for operating the licorice machine. For example, in one of the procedures it was explained how the sensors had to be programmed. The second version of the instructions (PS) contained the same 11 procedures and 4 additional blocks of system information. These blocks clarified the internal working of the machine. For example, in one of the blocks, it was explained how the sensors influenced the machine's regulators. The third version of the instructions (PSU) contained the same information as the PSinstructions and 3 additional blocks of utilization information. For example, in one of these blocks it was explained what the operator could do to make the licorice softer or more shining.

The user instructions were presented on the same computer screen as the control panel. If the space bar was pressed, the control panel disappeared from the screen and the content list of the instructions was shown. When the participants clicked on a section title, the corresponding text block was shown. By clicking on another title, the earlier block disappeared. The participants could return to the control panel by clicking on a 'return to the device' button. If they returned to the instructions later, the last activated information block was still visible.

To measure the performance of the participants, 12 different tasks were formulated. Task 1-6 required only procedures that were described in the procedural part of the user instructions. Task 7-12 required to infer steps that were not given explicitly in the instructions, but that could be derived easily from the system information in the PS- and PSU-versions of the instructions.

To measure the self-efficacy of the participants, a questionnaire was constructed. 3 questions were related to tasks that required only procedural information, 3 other questions were related to tasks that required a combination of procedural and system information, 3 other questions were related to tasks that required a combination of procedural, system, and utilization information. The participants had to rate their confidence that they could perform the different tasks on seven-point Likert scales. They could rate from 'Yes, I'm sure I can' to 'No, I'm sure I cannot'.

\subsection{Procedure and design}

Five to twelve participants worked simultaneously in a computer room, each on a separate PC. They were randomly assigned to one of the versions of the instructions when they entered the room. The only independent variable in this experiment was the version of the instructions.

After' a short introduction, the participants had to practice with the device for 30 minutes. During practicing, they could consult the instructions whenever they wanted. They had to rate their cognitive load after each five minutes. After 5, 10, 15, 20, and 25 minutes of practicing a small window appeared on the screen with the question 'How difficult is learning to work with the GDPC 324 at this moment?' The participants could rate their answers from 'extremely easy' to 'extremely difficult' on nine-point Likert scales that also appeared on the screen.

After practicing for half an hour, the participants had to stop; the questionnaire was handed to them. It took them approximately 10 minutes to answer the questions.

Next, the participants had to complete the 12 tasks. They could no longer consult the instructions during task execution. They had to decide themselves when they had finished the task and could start with the next; the program did not give them any feedback about whether they completed the task correctly. All actions that the participants performed while executing the tasks were logged automatically. The logfiles had to be analyzed later to examine whether the tasks were correctly executed and to calculate how much time was needed to complete the tasks. After each 3 questions, cognitive load was measured. The participants had to answer the question 'How difficult were the last couple of tasks?' on the same nine-point Likert scales that were used during practicing.

The participants needed 10 to 20 minutes to complete all tasks. The complete experiment took approximately an hour. 
cognitive load practicing

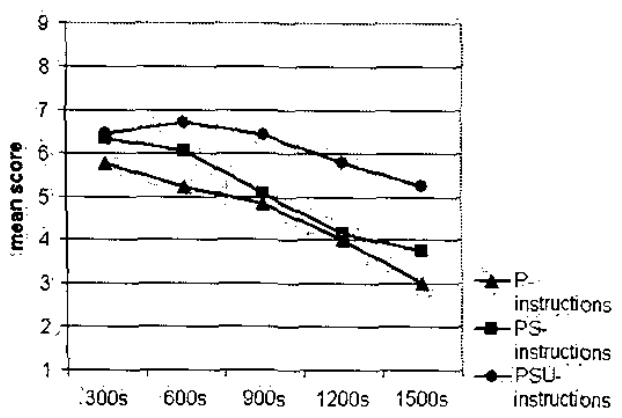

Figure 1. Mean cognitive load scores after 300, 600, 900,1200 , and 1500 seconds of practicing.

\section{Results}

The two types of declarative information did not have strong effects on task performance. Analyses of variance did not show significant differences in the number of tasks that were not completed correctly ( $F$ $(2,62)<1)$ nor in the time that was needed to complete each of the 12 tasks $(F(2,765)=1.53 ; p>0.20)$. However, when considering only the tasks that were completed correctly, a significant difference was found: $F$ $(2,603)=3.04 ; \mathrm{p}<0.05$. Tukey's HSD demonstrated that the participants who used PS-instructions needed less time to complete a task correctly than participants who used P-instructions $(p=0.05)$. The time that the participants who used PSU-instructions needed to complete a task correctly did not differ significantly from the two other groups of participants.

The presence of declarative information in the instructions did affect the cognitive load scores during practicing (see figure 1). The scores of all three groups of participants showed a comparable pattern; they decreased during practicing. The scores of the participants who used PSU-instructions were the highest on all moments in time, the scores of the participants who used PS-instructions were lower and the scores of the participants who used P-instructions were the lowest on all moments in time. Analyses of variance followed by Tukey's HSD tests demonstrated that the users of the PSU-instructions rated their cognitive load significantly higher than the users of the other two instructions after $10,15,20$ and 25 minutes of practicing $(F(2,62)>4.5$; $\mathrm{p}<0.05$ for all analyses of variance; $\mathrm{p}<0.05$ for Tukey's tests). No differences were found after $5 \mathrm{~min}$ utes of practicing: $(F(2,62)=1.06 ; p>0.30$.

\section{cognitlve load task performance.}

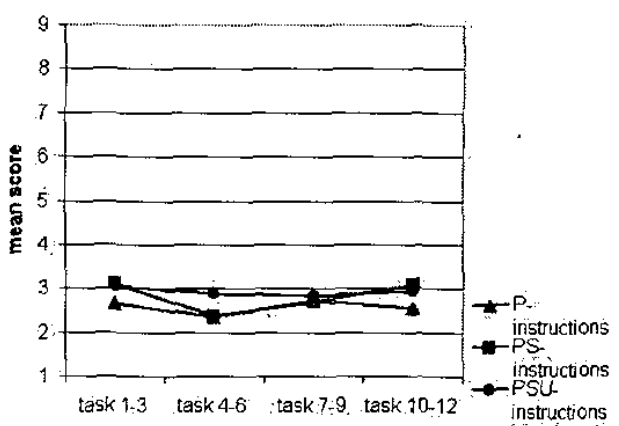

Figure 2. Mean cognitive load scores after completing $3,6,9$, and 12 tasks.

The presence of declarative information did not affect the cognitive load scores during task performance (see figure 2). No significant differences between the scores of the three groups of participants were found. There was no decrease or increase in the ratings and all participants considered the tasks rather easy to perform; the mean scores of the participants were between 2.4 and 3.1. Remember that they rate their cognitive load from 1 (very easy) to 9 (very difficult).

The self-efficacy scales used in the questionnaire proved to be sufficiently reliable. This holds for the complete scale reliability $(\alpha=0.89)$, as well as for the subscales for self-efficacy related to different types of tasks $(\alpha>0.70)$. The two types of declarative information in the instructions did affect the self-efficacy scores (see figure 3). An analysis of variance demonstrated significant differences in the confidence about the ability to perform tasks in general $(F(2,62)=3.67$; $\mathrm{p}<0.05)$. Tukey's HSD test demonstrated that participants who used the P-instructions were more con-

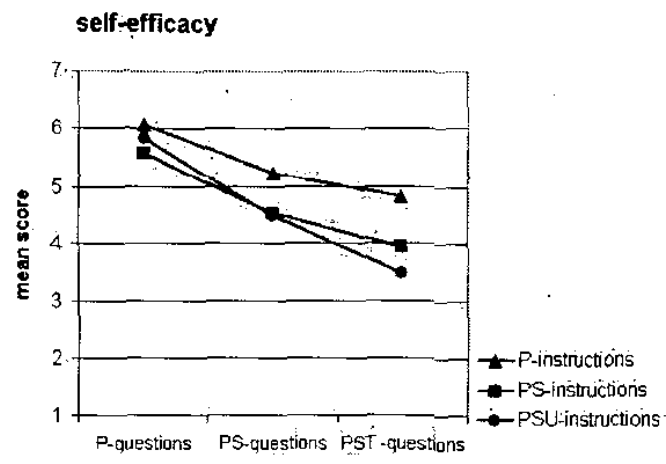

Figure 3. Means for self-efficacy ratings for P-, PS-, and PSU-instructions. 
fident than participants who used PSU-instructions ( $p<$ 0.05 ). Another analysis of variance demonstrated significant differences in the confidence in the ability to perform tasks that required a combination of procedural, system and utilization information $(\mathrm{F}(2,62)=7.16 ; \mathrm{p}<$ 0.01 ). Tukey's HSD test demonstrated that participants who used the P-instructions were more confident than participants who used PS-instructions $(p<0.05)$ and than participants who used PSU-instructions $(\mathrm{p}<0.01)$.

\section{Conclusions}

The results show that the presence of declarative information in instructions for use is an extra burden for users who have to work with a technical device. System information affected task performance moderately positively. However, this effect did not occur when utilization information was added to the other two types of information. Clearly, more research is needed to investigate the effects of different types of information on task performance; the results of different experiments are not conclusive.

The main objective of this experiment was to explore some other effects of system and utilization information. It was expected that reading system and utilization information would make the users more confident that they will be able to carry out different types of tasks. On the contrary, the results demonstrated that adding one or two types of declarative information decreased the self-efficacy ratings of the participants. A possible explanation for this effect might be that the participants with the P-versions underestimated the complexity of the device, while the $\mathrm{S}-$ and $\mathrm{U}$ information made the other participants aware of the complexity of the device by reading declarative information. As a result, confidence decreased.

The results of the cognitive load measurements demonstrated that adding one or two types of declarative information to procedural information results in increased mental effort during practicing. This effect was expected because the participants who used PS- or PSUinstructions read more information to build a more elaborate mental representation. It was expected that these efforts would be 'paid back' during task performance because at that moment, the elaborated mental representation would be useful. Therefore, it should be easier to perform the tasks. However, no differences in cognitive load scores were measured during task performance. All participants considered the tasks as rather easy. It might be that the tasks were too easy to be able to benefit from a more elaborated mental representation.

The effects of declarative information on selfefficacy and on cognitive load were never examined before. Therefore more research is needed before it can be concluded with certainty that instructions for use should not contain system and utilization information, but the results of this experiment indicate that these two types of information are not extremely useful.

\section{References}

[1] D. E. Kieras, and S. Bovair, "The role of a mental model in learning to operate a device," Cognitive science, vol. 8, pp. 255-273, 1984.

[2] P. Dixon, K. Harrison, and D. Taylor, "Effects of sentence form on the construction of mental plans from procedural discourse," Canadian Journal of Experimental Psychology, vol. 47, pp. $375-400,1993$.

[3] P. A. Bibby, and S. J. Payne, "Internalization and the use specifity of device knowledge," $\mathrm{Hu}$ man-computer interaction, vol. 8, pp. 25-56, 1993.

[4] M. M. N. Ummelen, Procedural and declarative information in software manuals. Effects on information use, task performance and knowledge. Amsterdam/Atlanta: Rodopi, 1997.

[5] P. Dixon, and G. Gabrys, "Learning to operate complex devices: Effects of conceptual and operational similarity," Human Factors, vol. 33, pp. 103-120, 1991

[6] P. Dixon, C. Zimmerman, and S. Neary, "Prior experience and complex procedures," Memory and cognition, vol. 25, pp. 381-395, 1997.

[7] J. Karreman, and M. Steehouder, "Het instellen van de Vox New York. Gebruik en effect van declaratieve informatie in gebruiksaanwijzingen [Operating the Vox New York. Use and effect of declarative information in instructions for use]", in Over de grenzen van de taalbeheersing: onderzoek naar taal, tekst en communicatie ( $\mathrm{R}$. Neutelings, N. Ummelen and A. Maes, Eds.). Sdu Uitgevers, Den Haag, 2000, pp. 273-283.

[8] J. Sweller, J. J. G. van Merriënboer, and F. G. W. C. Paas, "Cognitive architecture and instructional design," Educational Psychology Review, vol. 10, pp. 251-296, 1994.

[9] A. Bandura, "Social cognitive psychology: an agentic perspective," Annual review of psychol$o g y$, vol. 52, pp. 1-26, 2001. 
The Shape of Knowledge

\section{About the Authors}

Joyce Karreman holds a MA in Dutch linguistics at the University of Nijmegen. As a Ph.D. student at the University of Twente she teaches technical communication and document design, and she investigates the effects of declarative information in instructions.

Michaël Steehouder holds the chair of Technical Communication at the University of Twente. He is also chair of the Dutch society for Technical Communication STIC and board member of Tceurope. His research interests include the design of government forms and user instruction, and thetorical aspects of technical communication. He is an Associate Editor of IEEE Transactions on Professional Communication. 